\title{
Conceptual Framework for Surface Manager on Interactive Tabletops
}

Nur Alhuda Hamdan RWTH Aachen University 52056 Aachen, Germany

nur@cs.rwth-aachen.de

Jan Borchers

RWTH Aachen University

52056 Aachen, Germany

borchers@cs.rwth-aachen.de

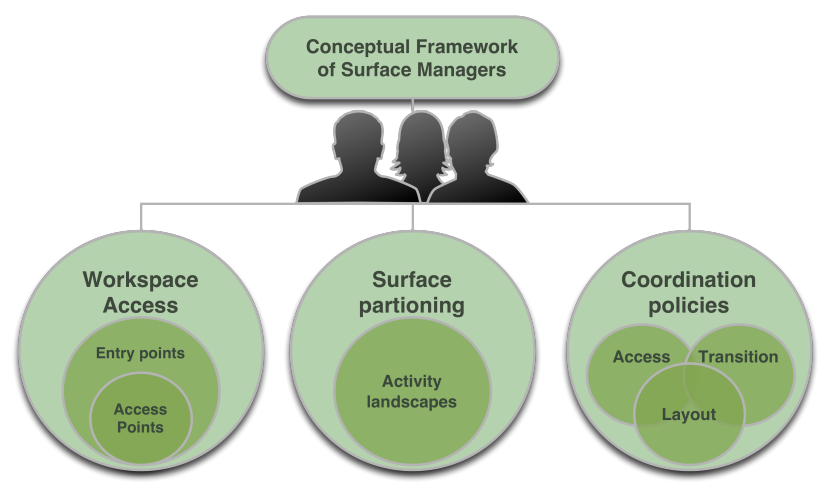

Figure 1: Conceptual framework of surface managers.

Copyright is held by the author/owner(s).

CHI 2013 Extended Abstracts, April 27-May 2, 2013, Paris, France.

ACM 978-1-4503-1952-2/13/04.
Simon Voelker

RWTH Aachen University

52056 Aachen, Germany

voelker@cs.rwth-aachen.de

\author{
Abstract \\ To date, most tabletop systems are designed with only a \\ single application visible and accessible at any time, which \\ is, in many cases, an underuse of the tabletop spacious \\ surface, and counter-intuitive to the normal working \\ environment of a table. Desktop window managers \\ provide users facilities to launch and interact with \\ concurrent applications, as well as manage their work \\ items. However, these managers are designed for \\ single-user systems and cannot be directly utilized in \\ tabletops without sacrificing usability. In our research, we \\ want to bring window manager facilities to tabletops. We \\ approach this by first constructing a conceptual \\ framework based on workplace theories and tabletop \\ investigations to understand how users structure their \\ work in these environments (see Figure 1). We will then \\ use the resulting framework to guide our design of a \\ sample surface manager.
}

\section{Author Keywords}

Interactive tabletop; window manager; conceptual framework; access points; entry points; coordination policies; territory.

\section{ACM Classification Keywords}

H.5.2 [Information interfaces and presentation]: User Interfaces. 


\section{Introduction}

Interactive tabletops form a unique class of computing devices. They offer a horizontal surface which affords social interaction, provides unconstrained display orientation, and allows placing physical artefacts on it. In addition, their spacious surfaces can positively influence work dynamics by allowing more natural and direct content manipulation and extending the visibility of the work space [9]. Yet, most design and interaction on these surfaces is limited to the single-application paradigm, i.e., only a single application is visible and accessible to the user at any time. This paradigm has several disadvantages in tabletop environments. Three of these disadvantages are:

- It constrains the user's interaction to a single application at a time, and requires her to remember the other running activities and how to switch between them.

- It limits the parallelism of co-located users.

- It can lead to misuse of the surface's size.

In this paper, we suggest that tabletop surfaces can be better exploited once we break away from the currently predominant single-application design paradigm, and provide structures and policies to support concurrent application interaction. For instance, for large surfaces to effectively engage spatial cognition and perception of multiple tasks we need to support space management mechanisms.

On classical desktop systems, existing window managers (e.g., Microsoft Windows and Apple's OS X) are designed for single-user systems with limited screen size, standard mouse and keyboard, support of a single control point, and fixed vertical orientation. Attempts to migrate window managers by merely scaling the interface and adapting the input modality can impose unnecessary limitations on tabletop interaction [10].

Mobile devices, such as tablets and smartphones, have the same direct-touch input modality as tabletops, but with limited screen space. On mobile devices the single-application design paradigm has proven successful, as it was utilized to account for the limited screen space and the mobile contexts-of-use. Currently, commercial tabletop systems, such as Microsoft Surface [1], implement window managers which resemble those of mobile devices in form and function. With only one application visible and responsive at any time, users are forced to continually switch between contexts. This is unnatural and counter-intuitive to the normal working environment of a table, where the user is able to view and interact with multiple pieces of information in parallel.

Our design process requires an understanding of how people work in interactive tabletop environments. However, little is understood and has been studied of the kinds of interactions parallel and collaborative users perform when attempting to accomplish several different tasks in a single session. The lack of standard tabletop technologies and long-term users led us back to more fundamental theories of workplace and tabletop territoriality. In particular, we build on Kirsh's model of the context of work [4]. Kirsh studied office workplaces to define an invariant structure of work that abstracts from superficial physical attributes, and is shared between various office settings. We extend his work with observations and empirical results from tabletop literature.

Thus, key contribution of this paper is a conceptual framework of the structure of work on tabletops, to guide the design of surface managers. 


\section{Related work}

Current tabletops are designed to support collaborative interaction within a single application. In a design effort to support application switching on tabletops, Ackad et al. [2] proposed Switch. Switch provides four functionalities: change application, switch between the set of files available for an application, alter application settings, and activate or deactivate interface elements within an application. In contrast, our goal is to support concurrent applications to run and be interactive simultaneously.

Two approaches have been taken to support running multiple applications on interactive tabletop surfaces. In [11], the authors suggest extending traditional window managers with multi-touch capabilities to enable researchers to transparently control user input and graphical output, simultaneously supporting both native multi-touch and single-pointer legacy applications. More recently, [12] developed uPlatform, a tool for creating customizable multi-user windowing systems on interactive tabletops. Although compelling, these previous approaches do not provide insights on user interaction and behavior in these environments, nor provide a coherent design for window managers on tabletops.

\section{Conceptual framework}

We are in the process of developing a descriptive model of the structure of work on tabletops. Our aim is to define a set of concepts to inform the design of a surface manager We focus on how to build a system that can support individuals and small groups to access digital resources, construct workspaces, and coordinate their actions on a horizontal surface. We are building a conceptual framework that extends Kirsh's model of the context of work that is based on the theoretical perspective of distributed cognition [4]. Our framework presents three concepts: workspace access, surface partitioning, and coordination policies.

Kirsh defines the context of work as the structure of informational, conceptual and physical resources that go beyond the superficial attributes of a work environment. Kirsh studied work environments from a cognitive science respective in order to understand the ecology and key components of a work environment deep structure and make them portable and abstract. He identified three key concepts that are shared among many work settings: entry points, activity landscapes, and coordination mechanisms (see Figure 2). In this paper, we apply, modify and extend Kirsh's model to co-located users in interactive tabletops environments.

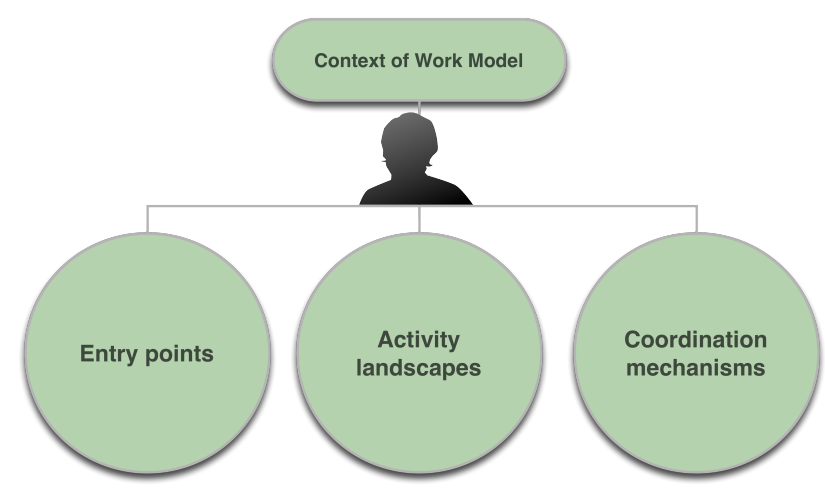

Figure 2: Kirsh's context of work model.

\section{Workspace Access}

A workspace is made up of many kinds of knowledge and structures, and the first part of the framework gives designers a basic idea of how to initiate an interaction with users. Entry points and access points are terms 


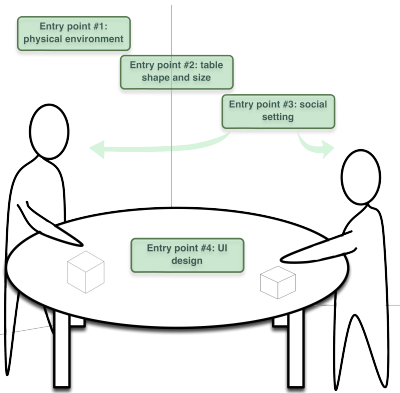

Figure 3: Entry points in a tabletop environment. which refer to the ways that the structure of an environment can mediate interaction with it $[4,3]$

\section{Entry points}

Entry points are cues and mechanisms that provide visibility, relevance, and overview of a space, and advance information about it $[3,4,5]$. In shared interfaces, entry points can contribute to the work context in terms of providing the users with a continuous perception of the state of digital and physical resources [7].

Lidwell et al. [5] describe entry points as one of the universal principles of design, and list three key features for them: minimal barriers, points of prospect, and progressive lures. Minimal barriers means allowing the user to access and move between entry points with minimum interference. On tabletops, an entry point that vaguely communicates its purpose or is hard to reach is one type of an undesired barrier. Other forms of barriers could be explicitly designed to prevent harmful actions.

Entry points should also provide points of prospect, that is, they must provide the user with enough time and space to review his options and understand the context. Visible and meaningful structures of entry points are one way to bring context to the user. For example, the flow, typeface and size of a newspaper's headlines provide the observer with information scent necessary to obtain a high-level conception of the content, and a rough plan to navigate through this information landscape [4].

Progressive lures means these points should be designed incrementally to guide the user to enter and move through the design. On interactive tabletops, the designer can offer a diverse set of incremental entry points to enable users to engage at different levels of interaction, gradually allowing mechanics of the systems to disappear, leaving the user with a sense of familiar and natural interaction with the content.

In interactive tabletop environments, entry points can be environmental, physical, social, or digital structures (see Figure 3). The physical environment around a tabletop is the first entry point the user encounters. The second entry point the user faces is the horizontal, relatively large and familiar surface of the table. Rogers et al. [7] found that the table ergonomics, i.e., size and shape, can play a role in luring people to approaching the surface. Other users who are already at the table can either have what Hornecker et al. [3] describe as the honey pot effect or discourage further approach, depending on the context. As the user finds space around the table or by merely observing others interact and experience the table, whatever design decisions the designer had made shape the primary entry points to the tabletop experience.

Access points

Access points denote characteristics that enable the user to actually interact and join a group's activity [3].

Tabletops are multi-touch surfaces which afford multiple concurrent users the option to access and actively manipulate relevant objects. This leads to the concept of multiple access points which essentially requires the system to be able to identify users.

We analyze the design of access points on tabletops from two dimensions: presentation and distribution. Several presentations of access points have been employed in tabletop design: graphical elements (e.g., menus and buttons), spatial locations, gestures, and physical objects (e.g., special tangibles, pens and smartphones). These presentations can be used in three distribution patterns: centralized, i.e., a set of access points is shared by all users; duplicated, i.e., a set of access points is duplicated 
around the borders of the shared table; and distributed, i.e., an arbitrary number of access point sets are located in arbitrary spatial locations over the tabletop surface.

On interactive surfaces, access points should be discoverable (e.g., by being labeled) and accessible (e.g., by distributing them closer to the table edges). A tabletop interface designer can design these access points to control the number of concurrent users on the surface at any time, the amount and type of accessible resources, and to impose access privileges. Access points also help increase awareness on the surface by indicating ownership of surrounding artefacts. A variety of input modalities and presentations can be used to identify access points' roles or ownership. Access points are cues and not content, and so they should have a limited footprint on the surface to reduce clutter.

\section{Surface Partitioning}

Several investigations of tabletop work practices have observed that users partition the surface into three different territories when performing activities to acquire resources and mediate group interactions: personal, group and storage territories [8]. Within these territories, users construct what Kirsh describes as activity landscapes, i.e. structures or environments that people build interactively while handling an activity. Each landscape has its own set of entry and access points, properties, and resources. A user composes a landscape from a collection of concepts, the layout of artefacts, his actions and consequences, and constrains imposed by a task or environment.

From tabletop territoriality research, we synthesized four aspects a designer should investigate when creating and partitioning territories: definition, properties, functionality, and policies. In Table 1, we present these aspects, and the related questions a designer should attempt to answer.

\begin{tabular}{|c|c|}
\hline Territ & Designer questions \\
\hline \multirow[t]{2}{*}{ Definition } & What kinds of territories to support. \\
\hline & $\begin{array}{l}\text { Which approach to adopt in dividing } \\
\text { and maintaining territories, i.e., de- } \\
\text { pend on users' seating arrangements } \\
\text { or table ergonomics. }\end{array}$ \\
\hline \multirow[t]{3}{*}{ Properties } & $\begin{array}{l}\text { What spatial properties each territory } \\
\text { has. }\end{array}$ \\
\hline & $\begin{array}{l}\text { Should the properties be dynamic or } \\
\text { fixed. }\end{array}$ \\
\hline & $\begin{array}{l}\text { What table properties affect the de- } \\
\text { signer's choice. }\end{array}$ \\
\hline Functionality & $\begin{array}{l}\text { What functionality should each terri- } \\
\text { tory provide. }\end{array}$ \\
\hline Policies & $\begin{array}{l}\text { What policies should each territory } \\
\text { implement. }\end{array}$ \\
\hline
\end{tabular}

Table 1: Aspects of surface partitioning.

\section{Coordination Policies}

Coordination policies are agents that facilitate manipulating objects and coordinating interaction. In offices, Kirsh describes the clock as one mechanism to facilitate time coordination between people. On traditional tables, social policies, i.e., standards of polite behavior, are used to coordinate people's interaction. However, on interactive tabletops, research shows that additional coordination policies on direct manipulation should be provided to coordinate access and solve conflicts [6]. For example, Morris et al. [6] describe how adult participants stole words from each other in a poem creation task with a tabletop interface. 
On interactive surfaces, we identify three kinds of coordination policies that should be supported:

- Layout polices to help increase workspace visibility, organize activity landscapes, exploit spacial cognition, and facilitate artefacts organizing.

- Access control policies to enable flexible access to table resources and workspaces, as well as to implement privacy settings.

- Transition policies to achieve fluid transitions between individual work and active collaboration, and to facilitate content sharing.

\section{Future work}

We are in the process of refining the conceptual framework and implementing a prototype of surface manager. We will conduct user studies to evaluate our design and detect any dispensary between the conceptual framework and the users' practices. Finally, we intend to explore how surface managers can lead to the emergence of new roles for interactive tabletops.

\section{References}

[1] Microsoft. Experience Microsoft Surface, available at: http://www.microsoft.com/surface/, Dec. 2012.

[2] Ackad, C., Collins, A., and Kay, J. Switch: exploring the design of application and configuration switching at tabletops. In Proc. ITS '10, ACM (2010), 95-104.

[3] Hornecker, E., Marshall, P., and Rogers, Y. From entry to access: how shareability comes about. In Proc. DDPI '07, ACM (2007), 328-342.

[4] Kirsh, D. The context of work. Human-Computer Interaction 16, 2-4 (2001), 305-322.

[5] Lidwell, W., Holden, K., and Butler, J. Universal Principles of Design, Revised and Updated: 125 Ways to Enhance Usability, Influence Perception,
Increase Appeal, Make Better Design Decisions, and Teach through Design. Rockport publishers, 2010.

[6] Morris, M., Ryall, K., Shen, C., Forlines, C., and Vernier, F. Beyond social protocols: Multi-user coordination policies for co-located groupware. In Proc. CSCW '04, ACM (2004), 262-265.

[7] Rogers, Y., Lim, Y.-k., Hazlewood, W. R., and Marshall, P. Equal opportunities: Do shareable interfaces promote more group participation than single user displays? Human-Computer Interaction 24, 1-2 (2009), 79-116.

[8] Scott, S. D., Sheelagh, M., Carpendale, T., and Inkpen, K. M. Territoriality in collaborative tabletop workspaces. In Proc. CSCW '04, ACM (2004), 294-303.

[9] Shen, C., Ryall, K., Forlines, C., Esenther, A., Vernier, F., Everitt, K., Wu, M., Wigdor, D., Morris, M., Hancock, M., et al. Informing the design of direct-touch tabletops. Computer Graphics and Applications, IEEE 26, 5 (2006), 36-46.

[10] Wang, X., Ghanam, Y., and Maurer, F. From desktop to tabletop: Migrating the user interface of agileplanner. Engineering Interactive Systems (2008), 263-270

[11] Wimmer, R., and Hennecke, F. Everything is a window: Utilizing the window manager for multi-touch interaction. In Proc. EICS '10 (2010)

[12] Wu, C., Suo, Y., Yu, C., Shi, Y., and Qin, Y. uPlatform: a customizable multi-user windowing system for interactive tabletop. Human-Computer Interaction. Design and Development Approaches (2011), 507-516 